\title{
La acción sustantiva universitaria y la articulación con el entorno: caso Sede Regional Chorotega de la Universidad Nacional de Costa Rica
}

\author{
The Substantive University Action and the \\ Integration with the Environment: The Case of \\ the Chorotega Regional Campus of the National \\ University of Costa Rica
}

\author{
Andrea Suárez Serrano ${ }^{l}$ \\ Universidad Nacional \\ Guanacaste, Costa Rica \\ andrea.suarez.serrano@una.cr \\ Sandra Lezcano Calderón ${ }^{2}$ \\ Universidad Nacional \\ Guanacaste, Costa Rica \\ sandra.lezcano.calderon@una.cr \\ Pável Bautista Solís ${ }^{3}$ \\ Universidad Nacional \\ Guanacaste, Costa Rica \\ pavel.bautista.solis@una.cr
}

Recibido 30/01/2018 Aceptado 06/04/2018

1 Centro Mesoamericano de Desarrollo Sostenible del Trópico Seco (CEMEDE-UNA), Sede Regional Chorotega.

2 Sede Regional Chorotega, UNA.

3 CEMEDE-UNA, Sede Regional Chorotega. 
Revista Universidad en DiÁlogo • Vol. 8, N. ${ }^{\circ}$ 1, Enero-Junio, 2018, pp. 39-56

ISSN 2215-2849 • EISSN: 2215-4752

DOI: http://dx.doi.org/10.15359/udre.8-1.3

Resumen. Se realizó una revisión de los proyectos de extensión-investigación de la Sede Regional Chorotega (SRCH) de la Universidad Nacional de Costa Rica para fortalecer la interrelación extensión-investigación-docencia y su articulación con la región. El objetivo de este trabajo es analizar cómo los proyectos de extensión e investigación influyen en la articulación interna y externa de la SRCH para cumplir con las diferentes actividades de la acción sustantiva universitaria. Se revisaron veintiocho proyectos de extensión-investigación, periodo 2012- 2017, utilizando un metaanálisis cualitativo y exploratorio de los proyectos registrados en el Sistema de Información Académica de la UNA. Los proyectos de extensión-investigación han fomentado una fuerte articulación de la UNA con comunidades y aliados estratégicos, ampliando el conocimiento de diversas disciplinas. Los proyectos permiten a las personas académicas mejorar su interacción y colaboración con organizaciones sociales, instituciones públicas y pares internacionales. Sin embargo, también es necesario revisar las políticas de regionalización para facilitar una mayor articulación con el sector privado y con otras universidades. Los fondos concursables regionales son esenciales para el desarrollo de proyectos de extensión e investigación. Sin estos recursos el alcance de las sedes regionales se reduciría drásticamente.

Palabras clave: articulación, cooperación, Región Chorotega, extensión universitaria, innovación.

Abstract. We reviewed the university extension and research projects of the Chorotega Regional Campus (SRCH) of the National University of Costa Rica (UNA) to strengthen the interrelation between extension, research, and teaching and their integration with the region. The objective of this paper is to analyze how the university extension and research projects influence the internal and external integration of SRCH to accomplish the activities of the substantive university action. Twenty-eight extension and research projects were reviewed; SRCH ran them from 2012 to 2017. The review was conducted using a qualitative and exploratory metaanalysis of the projects recorded in the UNA Academic Information System. The extension-research projects have encouraged a strong integration of UNA with communities and strategic allies, expanding the knowledge of diverse disciplines. The projects allow professors to improve their interaction and collaboration with social organizations, public institutions, and international peers. However, it is also necessary to review the regionalization policies to facilitate a greater integration with the private sector and other universities. The regional grant funds are essential to develop extension-research projects. Without such grants, the outreach of the regional campuses will be drastically reduced.

Keywords: integration, cooperation, Región Chorotega, extension, innovation. 


\section{Introducción}

\section{Descentralización universitaria y la historia de la SRCH.}

La educación universitaria de Costa Rica siguió el patrón de centralización característico del resto de la institucionalidad pública de este país. Las principales universidades públicas: Universidad de Costa Rica (UCR), Universidad Nacional de Costa Rica (UNA) y el Instituto Tecnológico de Costa Rica (TEC) fueron establecidas en sedes ubicadas en el Valle Central, desde donde por décadas se encargaron de facilitar su acción sustantiva. Este modelo representa desde luego grandes retos para las poblaciones rurales, especialmente aquellas de las provincias con los mayores desafíos en desarrollo humano, como las provincias de Puntarenas, Limón y por supuesto Guanacaste. Las familias rurales de estas provincias se encontraban con mayores dificultades para acceder al sistema de educación universitaria, dado que esto implica una mayor inversión en traslados, vivienda y alimentación.

Ante esta situación, el Estado costarricense planteó la Política de Regionalización Universitaria. Esta consistía en establecer sedes regionales en zonas rurales del país, facilitando que los estudiantes de estas zonas pudieran acceder con menores costos a una educación universitaria de calidad en provincias alejadas del Valle Central. La UCR fue pionera de este proceso al establecer el Primer Centro Regional en San Ramón de Alajuela y Liberia Guanacaste en 1968 (Castro, 2009). Posteriormente, la UNA, desde sus inicios en 1973, estableció Secciones Regionales que posteriormente se convirtieron en Sedes Regionales (Schmidt, 2016). Este es el caso de la Sede Regional Chorotega (SRCH), que desde 1998 adquirió esta condición, ubicada en la provincia de Guanacaste al noroeste de Costa Rica.

La SRCH, al igual que la UNA, ha evolucionado para poder responder a los retos y demandas de la sociedad. Varios autores han señalado que, ante las limitaciones de recursos del estado, las universidades públicas en Costa Rica han venido expandiendo su acción sustantiva para apoyar actividades más allá de la docencia como la extensión universitaria. Para cumplir su acción sustantiva la UNA establece en su Estatuto Orgánico cuatro actividades definidas: la docencia, la investigación, la extensión y la producción (UNA, 2016). Por ejemplo, la definición oficial del proceso de extensión es descrito en los lineamientos para la gestión de programas, 
Revista Universidad en DiÁlogo • Vol. 8, N. ${ }^{\circ} 1$, Enero-Junio, 2018, pp. 39-56

ISSN 2215-2849 • EISSN: 2215-4752

DOI: http://dx.doi.org/10.15359/udre.8-1.3

proyectos y actividades (PPAS) como “... procesos de creación y desarrollo de las capacidades de sus actores sociales, institucionales y locales, y de transformación social integral para una mayor calidad de vida de las comunidades" (UNA, 2016, p. 34).

El desarrollo de la extensión universitaria planteó nuevos retos para la UNA, ya que estas actividades demandan preparación, sensibilidad y recursos que tienen que competir con otras responsabilidades como la docencia, la investigación y la producción. A pesar de que la filosofía de la UNA y sus personas académicas ha sido enfocar esfuerzos para el desarrollo de las cuatro actividades mencionadas, los recursos son escasos y limitan la dedicación a cada una de estas actividades. Por ejemplo, un estudio identificó como causa del rezago en carrera académica de personas académicas propietarias a la poca participación en proyectos de extensióninvestigación y la dedicación a actividades que no son consideradas en la evaluación de carrera académica (Fernández, 2015).

El presente artículo tiene por objetivo analizar cómo los proyectos de extensión e investigación influyen en la articulación interna y externa de la SRCH para cumplir con las diferentes actividades de la acción sustantiva universitaria (Consejo Académico de la Universidad Nacional de Costa Rica, 2009).

A través de un metaanálisis cualitativo y exploratorio de los registros de proyectos liderados por la $\mathrm{SRCH}$, se describen las características de las fuentes de financiamiento de los proyectos y se analiza el impacto en la articulación. La pregunta de investigación general de este trabajo es: ¿Tiene algún efecto el desarrollo de proyectos de extensión-investigación liderados por la SRCH en la articulación universitaria con su entorno? Aunque este estudio se enmarca en el análisis de un caso de estudio de una Sede Regional de la UNA, los hallazgos facilitan la identificación de retos y lecciones valiosas para el mejoramiento del quehacer universitario de Costa Rica. Este trabajo da continuidad a los esfuerzos académicos de la SRCH para destacar su aporte a la sociedad y demostrar que la práctica puede informar y sentar las bases para la construcción de mejores políticas universitarias (Gamboa, Céspedes, Mena y Azofeifa, 2017; Maldonado, 2014; Monge, Lezcano y Méndez, 2014; Schmidt, 2016).

La estructura del artículo es la siguiente: a) Inicia con una breve introducción sobre la descentralización universitaria. b) Materiales y métodos, describiendo la 
estrategia de muestreo de los proyectos, los análisis cualitativos del metaanálisis, la definición dearticulación y el desarrollo de estadística descriptiva para presentar los principales resultados. c) Los resultados y discusión del metaanálisis, con énfasis en las características de los proyectos que influyen en la articulación y la cuantificación de la articulación interna y externa desarrollada en los proyectos de extensión-investigación. (d) Principales recomendaciones para mejorar la articulación y el desarrollo de la extensión-investigación en la SRCH.

\section{Materiales y métodos}

\section{Área geográfica del estudio}

La Sede Regional Chorotega de la Universidad Nacional de Costa Rica (SRCH) se encuentra ubicada en el noroeste de Costa Rica. Dos campus conforman esta sede: 1) Uno se encuentra ubicado en la ciudad de Nicoya; 2) mientras que el otro se encuentra en la ciudad de Liberia, ambos de la provincia de Guanacaste. En conjunto las dos sedes ofrecen una oferta académica de siete carreras y dos programas de investigación: a) En desarrollo sostenible a cargo del Centro Mesoamericano de Desarrollo Sostenible del Trópico Seco (CEMEDE); y b) en agua con el Centro de Recursos Hídricos para Centroamérica y el Caribe (HIDROCEC). El cuerpo académico está conformado por 133 personas académicas, que facilitan el aprendizaje de 1653 estudiantes, 904 del campus en Liberia y 749 del campus en Nicoya (Rojas, 2017). Todas las personas académicas de la UNA desarrollan su actividad sustantiva en torno a la extensión, la docencia, la investigación y la producción.

\section{Selección de proyectos y sistematización de la información}

La UNA utiliza a partir del año 2010 el Sistema de Información Académica (SIA) encargado de registrar todas las actividades formales de la UNA relacionadas con la gestión y administración de programas, proyectos y actividades académicas (Monge, Lezcano y Méndez, 2014). El SIA cuenta con un total de 3361 registros (a octubre de 2017), de los cuales como marco muestral se utilizaron 130 registros (3.9\%) desarrollados en la Sede Regional Chorotega (ver figura 1). Se utilizaron los siguientes criterios de selección para identificar los registros a analizar: 
Revista Universidad en DiÁlogo • Vol. 8, N. ${ }^{\circ}$ 1, Enero-Junio, 2018, pp. 39-56

ISSN 2215-2849 • EISSN: 2215-4752

DOI: http://dx.doi.org/10.15359/udre.8-1.3

1) Registros desarrollados en la Sede Regional Chorotega.

2) Registros desarrollados en el periodo 2012-2017, se consideró este periodo porque a partir de 2012 la administración de los proyectos usando SIA fue homogénea.

3) Registros liderados por personas académicas de la SRCH.

4) Registros de tipo proyecto. De acuerdo al Estatuto Orgánico de la UNA (2016), un proyecto es "... un conjunto de actividades académicas con carácter disciplinario o multidisciplinario programadas en un lapso determinado que se realizan para conseguir ciertos objetivos" (p. 36).

5) Registros de tipo académico, excluyendo actividades de vinculación.

6) Registros cuyo estado actual sea concluido, por concluir, vigente o avalado.

Con estos criterios se identificó la muestra del estudio conformada por un total de 28 registros del SIA $(0.83 \%$ del total de registros del SIA, $21.5 \%$ del total de los 130 registros de la Sede Regional Chorotega).

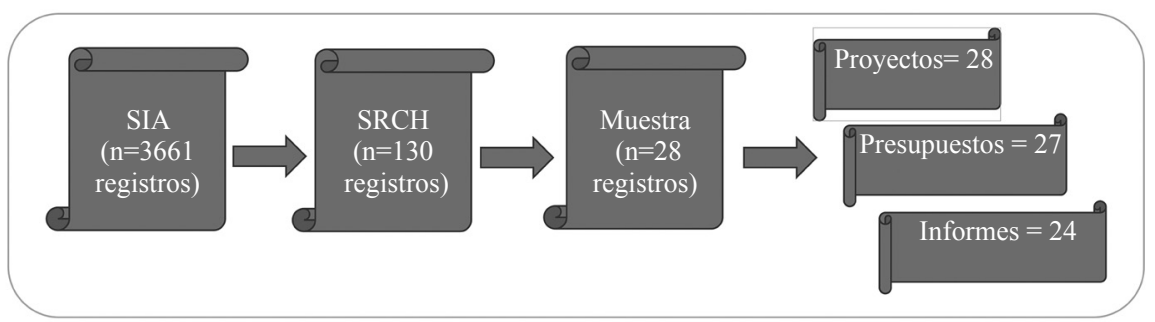

Figura 1. Esquema del muestreo e identificación de fuentes de información

De cada registro del SIA se obtuvo su propuesta de formulación $\left(\mathrm{n}_{1}=28\right)$, presupuesto $\left(\mathrm{n}_{2}=27\right)$ e informes parciales $\left(\mathrm{n}_{3}=16\right)$ y finales $\left(\mathrm{n}_{4}=8\right)$ disponibles. Todos los documentos ( $\mathrm{n}=79$ documentos) fueron digitalizados y exportados a un procesador de texto para verificar la ortografía de toda la información obtenida. Posteriormente, se importaron los documentos al software de análisis cualitativo NVivo 11 (QSR International, 2017). Además, se categorizó el contenido de los proyectos para facilitar su descripción y clasificación, especialmente para identificar y cuantificar las articulaciones (ver tabla 1). 
Tabla 1

Criterios para definir el tipo de articulación desarrollado en los proyectos liderados por la Sede Regional Chorotega de la Universidad Nacional de Costa Rica

\begin{tabular}{|c|c|c|}
\hline Número & Tipo de articulación & Descripción \\
\hline 1 & Intrainstitucional & $\begin{array}{l}\text { Proyectos con participantes de otras unidades } \\
\text { académicas de la Universidad Nacional. }\end{array}$ \\
\hline 2 & Interuniversitaria & $\begin{array}{l}\text { Proyectos con participantes que laboran } \\
\text { en otras universidades públicas o privadas } \\
\text { nacionales. }\end{array}$ \\
\hline 3 & Interinstitucional & $\begin{array}{l}\text { Proyectos con participantes que laboran en } \\
\text { instituciones públicas nacionales. }\end{array}$ \\
\hline 4 & Organización social & $\begin{array}{l}\text { Proyectos con participantes de } \\
\text { organizaciones sociales (cooperativas, } \\
\text { asociaciones, fundaciones, entre otras ) }\end{array}$ \\
\hline 5 & Sector productivo & $\begin{array}{l}\text { Proyectos con participantes del sector } \\
\text { privado productivo nacional. }\end{array}$ \\
\hline 6 & Internacional & $\begin{array}{l}\text { Proyectos con participantes que laboran en } \\
\text { universidades y centros de investigación del } \\
\text { extranjero. }\end{array}$ \\
\hline
\end{tabular}

Nota: Para ser cuantificada esta participación debe estar registrada en algún documento del proyecto.

La articulación es un concepto usado frecuentemente en la institucionalidad costarricense. Sin embargo, no se han encontrado referencias académicas sobre el significado de este concepto en el contexto de la extensión o regionalización universitaria de Costa Rica. Las referencias existentes mencionan el concepto sin describir su significado. En la práctica de hecho el concepto de articulación se utiliza como un sinónimo de coordinación. Por tanto, a continuación, se revisan las diferentes acepciones del concepto encontradas en varios textos y se adopta una definición operativa para el presente estudio.

El Estatuto Orgánico describe la necesidad de articular el desarrollo de la acción sustantiva en congruencia con las políticas universitarias y el contexto regional (UNA, 2016). Asimismo, Maldonado (2014) indica que el Plan Quinquenal 2006-2010 del Consejo Nacional de Rectores 
Revista Universidad en DiÁlogo • Vol. 8, N. ${ }^{\circ}$ 1, Enero-Junio, 2018, pp. 39-56

ISSN 2215-2849 • EISSN: 2215-4752

DOI: http://dx.doi.org/10.15359/udre.8-1.3

(CONARE) tiene como meta “... potenciar las capacidades del conjunto de las universidades públicas mediante la unión de esfuerzos a partir del mejor aprovechamiento de las fortalezas de cada institución". Por otro lado, el Plan Quinquenal de CONARE 2011-2015 menciona que la articulación es un mecanismo para establecer vínculos entre las actividades sustantivas; y confirma que su propósito es aprovechar el legado de cada universidad pública para el uso racional de los recursos (CONARE, 2011).

Esta articulación, según el CONARE (2008), debería ser entre universidades y con otros actores de la sociedad partiendo de necesidades consensuadas para maximizar los recursos propios, fortaleciendo las actividades existentes para el mejoramiento de las condiciones de vida de los habitantes de las zonas periféricas. Además, CONARE considera que la articulación se encuentra en un plano superior al de la coordinación, ya que provoca mayores beneficios en las regiones con bajos índices sociales.

Considerando lo anterior, esta ponencia utilizará la definición de articulación de Zúñiga y Lezcano (2015), quienes la conciben como:

La asociación de dos o más instituciones que planifican acciones en forma cooperativa para desarrollar una acción en forma conjunta. Estas acciones están incorporadas dentro de la planificación institucional de cada una de las partes e incluye el compromiso de inversión de recursos institucionales (financieros, humanos, materiales) para su obtención y su no cumplimiento por una de las partes afecta la posibilidad del logro de la acción para la otra parte. (p. 81)

Se utilizó un enfoque de métodos mixtos para el análisis de la información. Primero, se registraron en una hoja de cálculo los datos cuantitativos y de clasificación de los PPAS y esta información se analizó utilizando estadística descriptiva y la librería ggplot2 (Wickham, 2009) y extracat (Pilhöfer y Unwin, 2013) de R statistics (R Development Core Team, 2008). Adicionalmente, en Nvivo 11 se efectuó un análisis cualitativo, para identificar lecciones aprendidas relacionadas con la articulación entre personas académicas de la sede, con otros centros de la UNA y con socios externos. Asimismo, se construyó una línea de tiempo con factores relevantes para la articulación con timeline maker (TIMEGRAPHICS, 2017). 


\section{Resultados y discusión}

\section{Descripción general de los proyectos analizados}

Los veintiocho registros liderados por la Sede Regional Chorotega de la Universidad Nacional de Costa Rica pueden clasificarse en dos categorías de acuerdo al origen de su financiamiento (ver figura 2). a) Recursos internos de la UNA de las convocatorias: FIDA de la Vicerrectoría de Extensión $(\mathrm{n}=3)$; y FUNDER de la Vicerrectoría de Extensión $(\mathrm{n}=13)$. b) Fuentes de financiamiento externas: CONARE Regionalización del Consejo Nacional de Rectores de Costa Rica $(n=9)$ y Fondos Externos $(n=3)$ internacionales o nacionales (e.g. Horizonte 2020, CSUCA, entre otros). Todos los fondos son concursables, lo que implica competir con propuestas de calidad y que permitan atender las necesidades regionales y locales.

El presupuesto promedio y la duración de los proyectos muestran diferencias de acuerdo a la fuente de financiamiento. Los proyectos financiados por fondos externos $(n=3)$ tienen un mayor presupuesto promedio $(\bar{x}=40.45$ millones de colones, $\mathrm{DE}=13.32$ millones de colones); mientras que los fondos internos de la UNA tienen un presupuesto promedio menor al menos en un $50 \%$. Con respecto a la duración de los proyectos, FIDA tiene la mayor duración promedio ( $\bar{x}=37.56$ meses, $\mathrm{DE}=3.89$ meses $)$ y CONARE Regionalización la menor ( $\bar{x}=23.34$ meses, $\mathrm{DE}=8.25$ meses $)$.

Las diferencias en el origen del financiamiento para los proyectos liderados por la SRCH pueden estar relacionadas con los siguientes factores: 1) Los fondos FUNDER y CONARE Regionalización priorizan la participación de las sedes regionales y se enfocan en temas de extensión universitaria, que es una de las fortalezas de la SRCH. 2) Los fondos CONARE Regionalización son indispensables porque aportan recursos para el pago de salarios de las personas académicas y otras personas profesionales. 3) Aunque FIDA es una opción atractiva de financiamiento porque ofrece un presupuesto mayor al resto de los fondos internos de la UNA, el concurso FIDA es más competitivo y los temas financiados tienen un menor componente de extensión. 
Revista Universidad en DiÁlogo • Vol. 8, N. ำ 1 , Enero-Junio, 2018, pp. $39-56$

ISSN 2215-2849 • EISSN: 2215-4752

DOI: http://dx.doi.org/10.15359/udre.8-1.3
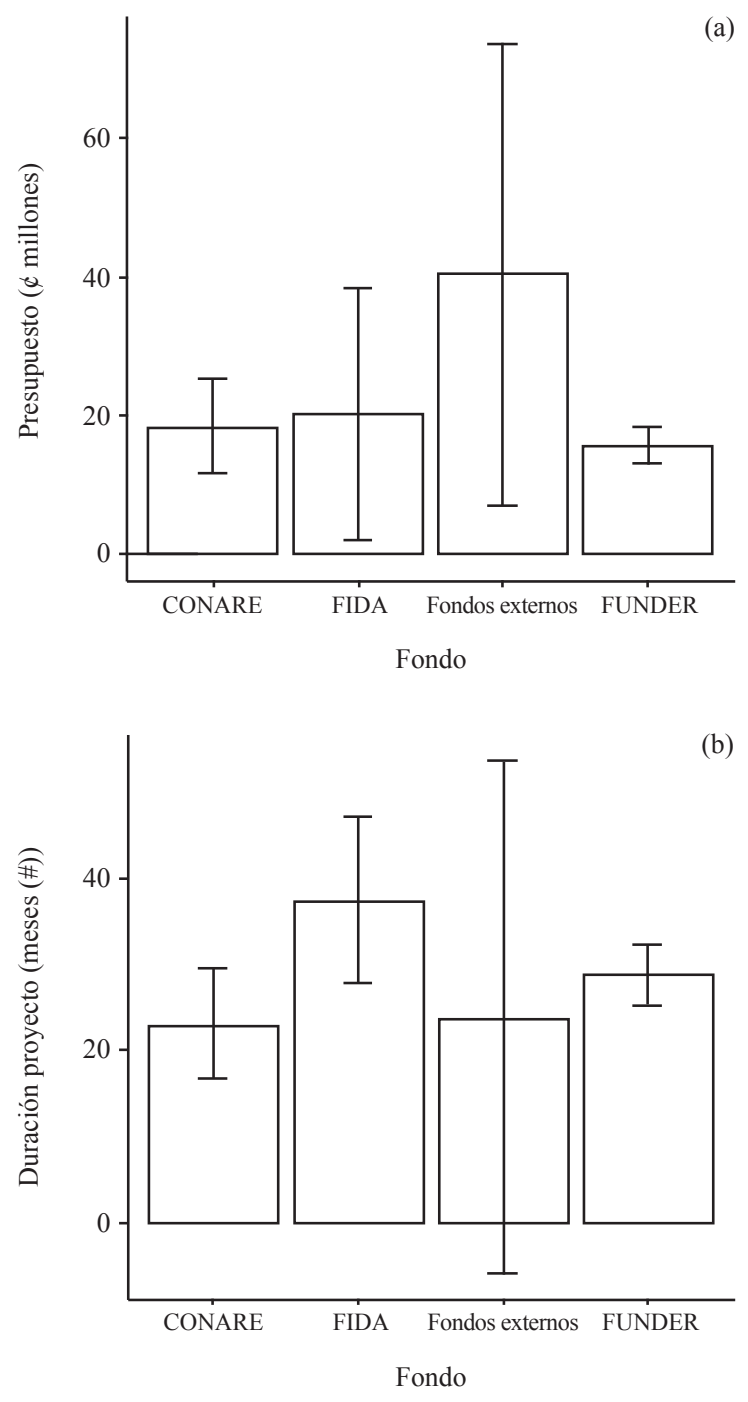

Figura 2. a) Presupuesto promedio 2012-2017 y b) duración promedio de los veintiocho proyectos liderados por la Sede Regional Chorotega, Guanacaste, Costa Rica.

La mayoría de los proyectos liderados por la SRCH han sido de carácter interdisciplinario, principalmente desarrollando actividades en el área académica de extensión y financiados por FUNDER y CONARE Regionalización (ver figura 3). Los proyectos desarrollados en el área académica de investigación 
son solo dos y estos han sido financiados por FIDA y Fondos Externos. Las actividades académicas integradas son transversales a todas las fuentes de financiamiento. La tendencia a desarrollar proyectos interdisciplinarios y multidisciplinarios puede explicarse por la estructura de los perfiles de las personas académicas de la $\mathrm{SRCH}$, que incluye profesionales de diversas disciplinas. Además, en esto también influyen los lineamientos de las fuentes de financiamiento que generalmente valoran mejor las propuestas multidisciplinarias e interdisciplinarias (CONARE, 2008; CONSACA 2009, 2016 y 2017).
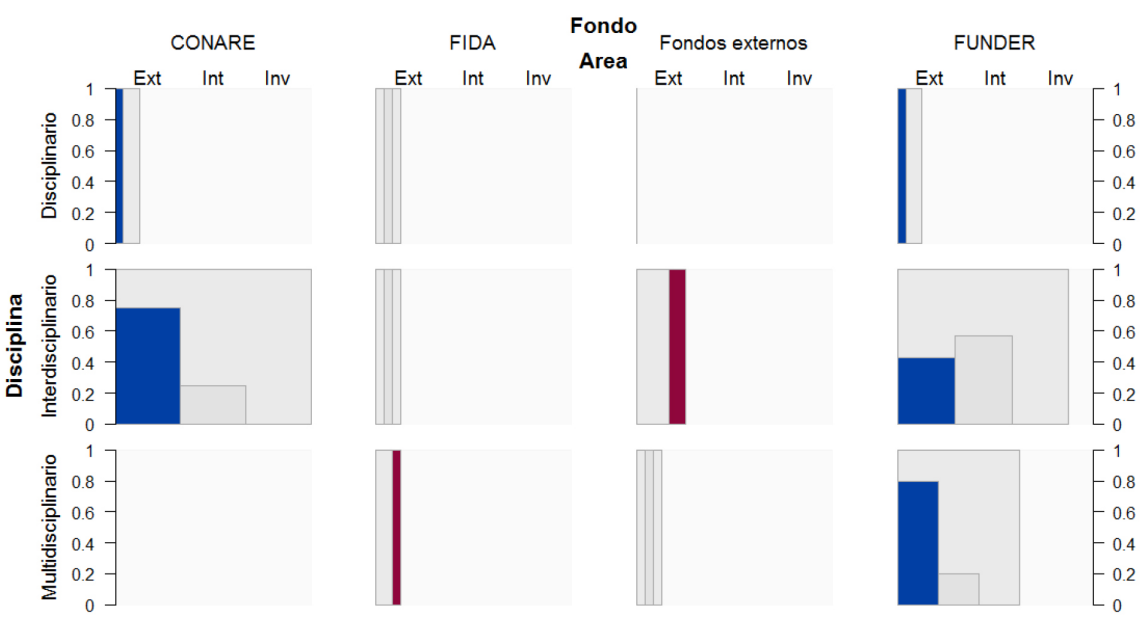

Figura 3. Fuente de financiamiento, disciplina y área académica de los veintiocho proyectos liderados por la Sede Regional Chorotega de la Universidad Nacional de Costa Rica en el periodo 2012-2017. Nota: las barras indican las frecuencias condicionales relativas en una tabla de contingencia de tres dimensiones. Áreas académicas: Ext= Extensión (color azul), Int= Integrado (color gris), Inv= Investigación (color rojo).

\section{Articulación en la acción sustantiva de la Sede Regional Chorotega}

El análisis realizado con los veintiocho proyectos seleccionados evidencia que hay una tendencia al incremento de la articulación entre unidades académicas de la UNA (intrainstitucional), con instituciones públicas de la región Chorotega (interinstitucional), organizaciones sociales, universidades e institutos de investigación internacionales (internacional). Además, se ha mantenido una relación constante con universidades públicas (interuniversitaria) en la región y se observó una menor articulación con el 
Revista Universidad en DiÁlogo • Vol. 8, N. ${ }^{\circ}$ 1, Enero-Junio, 2018, pp. 39-56

ISSN 2215-2849 • EISSN: 2215-4752

DOI: http://dx.doi.org/10.15359/udre.8-1.3

sector productivo privado (ver figura 4). Sin embargo, como se observa en la figura 4, la articulación con universidades públicas disminuyó a partir del 2012. Esta tendencia responde al cambio en los lineamientos de los fondos de regionalización de CONARE, los cuales ya no requieren formulación conjunta con otras universidades públicas a partir del 2013.
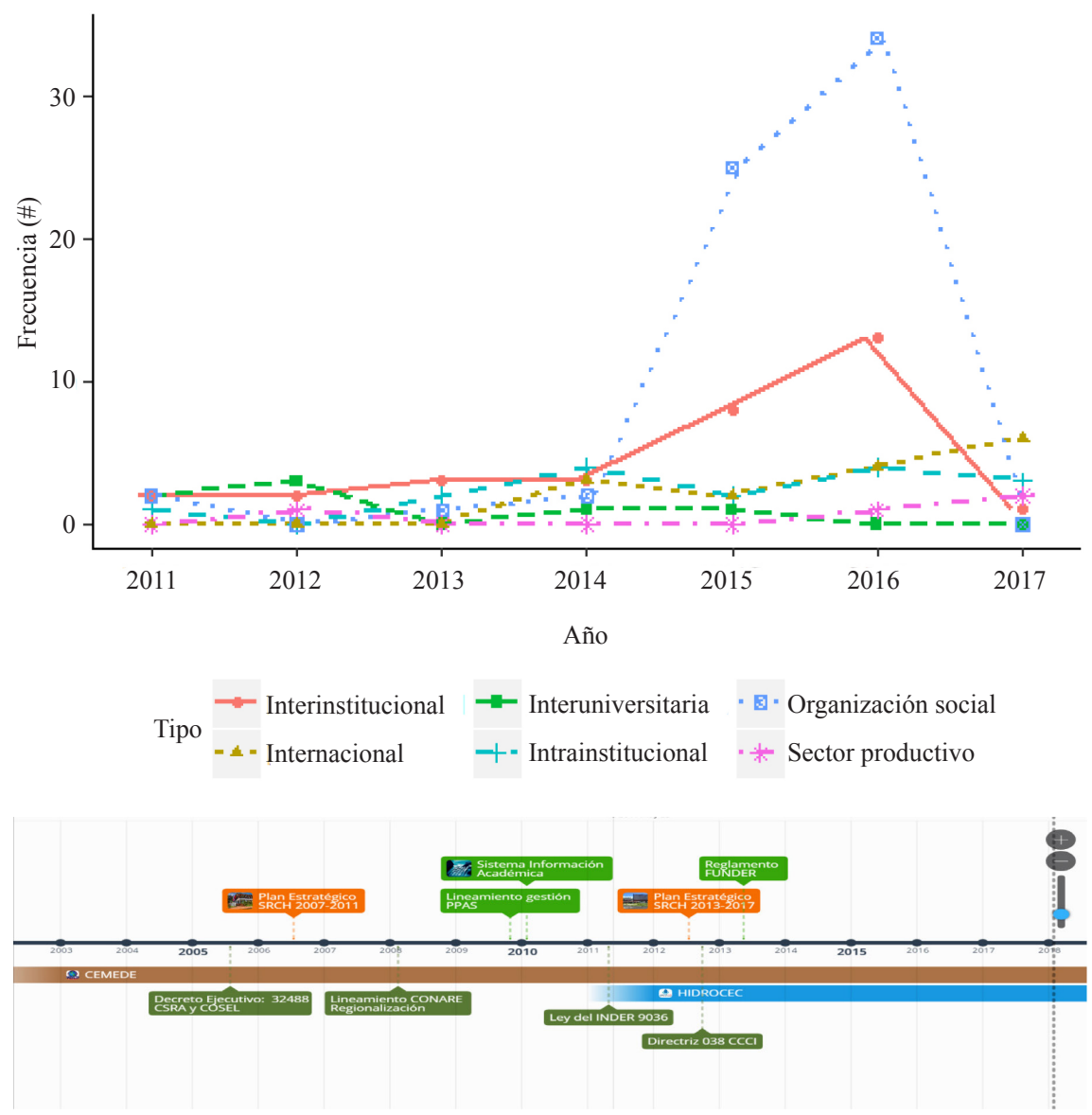

Figura 4. a) Número de colaboraciones en el periodo 2012-2018 con actores nacionales e internacionales por año y tipo de articulación. b) Eventos de relevantes para la articulación de la Sede Regional Chorotega, Guanacaste, Costa Rica. Nota: a) Las frecuencias indican la frecuencia de articulación anual y no son acumuladas. (b) Políticas SRCH (naranja); normativa costarricense (verde obscuro); políticas UNA (verde claro); programas de investigación SRCH (café y azul). 
La tendencia al aumento en la articulación con organizaciones sociales, a nivel interinstitucional e internacional obedece probablemente a la congruencia de un conjunto de políticas de coordinación interinstitucional, reformas en políticas de educación superior y lineamientos de proyectos, orientación estratégica de la SRCH y la experiencia y habilidad de personas académicas de la SRCH. Por ejemplo, el Estado costarricense ha estado promoviendo la coordinación interinstitucional a escala municipal y regional a través de los Comités Sectoriales de Locales (COSEL) y los Consejos Cantonales de Coordinación Interinstitucional (CCCI) (Poder Ejecutivo, 2005 y 2012). Además, la ley 9036 del INDER demanda la participación de personas académicas en espacios de coordinación para el desarrollo territorial (Asamblea Legislativa, 2012). Las personas académicas de la $\mathrm{SRCH}$ participan en estos espacios, permitiendo el fortalecimiento del capital social y el intercambio de información sobre necesidades y actividades de investigación y extensión, lo que ha dado resultados positivos que se reflejan en las formulaciones.

Por otro lado, la planeación estratégica de la UNA y la SRCH respaldan el desarrollo de proyectos de extensión e investigación contextualizados y que respondan a necesidades regionales. Específicamente, los dos últimos Planes Estratégicos de la SRCH de los periodos 2007-2011 y 20122017 promueven la articulación con unidades académicas de la UNA y universidades públicas. Adicionalmente, la contratación de personas académicas con experiencia en formulación y ejecución de proyectos y con contactos internacionales facilita la gestión de fondos externos.

La teoría institucional y organizacional puede ayudar a comprender por qué la articulación es deseable, pero también por qué existen dificultades para establecerla. En primer lugar, si la articulación es concebida como un proceso, es natural esperar que la institucionalidad y las personas necesiten comprender y adaptar sus reglas del juego y factores facilitantes. Además, es necesario adoptar una cultura adaptativa para valorar los objetivos comunes, en lugar de agendas particulares de una sola institución pública; esto ha sido documentado anteriormente en Costa Rica al describir el proceso del Programa de Regionalización Interuniversitaria (Lezcano, 2011). Asimismo, debe comprenderse que la articulación implica costos para todas las partes involucradas (Weiss, 1987). Si no existen recursos para cubrir la inversión en actividades conjuntas, difícilmente las instituciones públicas materializarán la articulación. En el caso de la $\mathrm{SRCH}$, los fondos para la implementación de proyectos proporcionan parte de estos recursos, por lo que estos tienen una influencia positiva en la articulación. 
Revista Universidad en DiÁlogo • Vol. 8, N. ${ }^{\circ}$ 1, Enero-Junio, 2018, pp. 39-56

ISSN 2215-2849 • EISSN: 2215-4752

DOI: http://dx.doi.org/10.15359/udre.8-1.3

Además, Weiss (1987) propone un modelopara la articulación entre instituciones públicas que requiere la existencia de una necesidad o problema complejo que pueda ser abordado a través de la articulación. Analizando la situación de la región Chorotega (escala local) y del corredor seco centroamericano (escala regional) es claro que los retos que está imponiendo el cambio climático y las limitantes en recursos económicos y tecnológicos sobre los medios de vida rurales pueden constituirse en problemas sumamente complejos que deben ser abordados de manera integral, colaborativa y adaptativa. Por otro lado, Weiss (1987) también señala la necesidad de contar con una capacidad institucional para poder desarrollar la articulación. Esta puede incluir siempre aspectos de legalidad y legitimidad que en la SRCH están siendo promovidos por las políticas internas y externas que fomentan la gobernanza colaborativa.

\section{Hacia una práctica de la extensión dialógica, articulada einnovadora}

La implementación de proyectos de investigación-extensión financiados con fondos concursables son un elemento esencial para la articulación con otras instancias del sector público, social y privado. La SRCH, al igual que la UNA y otras universidades públicas del país, cuenta con recursos limitados en su presupuesto institucional, estos difícilmente podrían comprometerse para ser invertidos en proyectos externos sin crear un desbalance presupuestario para otras actividades sustantivas. Consecuentemente, el presupuesto obtenido en concursos externos e internos facilita la articulación y la dedicación de otros recursos de la SRCH para favorecer el desarrollo regional.

La articulación intrainstitucional, interinstitucional y con organizaciones sociales no depende estrictamente de la cantidad de presupuesto y la duración asignada para su ejecución. Sin embargo, estos aspectos sí parecen influir en la articulación internacional y el alcance geográfico de los proyectos. El financiamiento externo de proyectos con un mayor componente de investigación beneficia el aumento de la articulación internacional con universidades e institutos de investigación internacional.

E1 Plan Estratégico de la Sede Regional Chorotega (2007-2011) tiene como objetivo estratégico fortalecer la vinculación externa y la cooperación nacional e internacional con diversas entidades públicas y privadas, con el fin de dinamizar el quehacer de la SRCH y contribuir más efectivamente al desarrollo local, regional y nacional, del cual se deriva la acción estratégica: 
desarrollo de trabajo conjunto con los actores de la sociedad civil y gubernamentales. También tiene como acción estratégica la realización de estudios o investigaciones, foros y otras actividades en temas de interés regional que potencian la articulación. Al mismo tiempo, el aumento en la articulación en el año 2012 coincide con la inauguración de las instalaciones de HIDROCEC. Aunado a esto, los fondos concursables promueven los diferentes tipos de articulación definidos en este estudio.

\section{Conclusiones}

El análisis exploratorio de los registros de veintiocho proyectos liderados por la Sede Regional Chorotega de la Universidad Nacional permitió demostrar la contribución de estas iniciativas a la acción sustantiva. Específicamente, este estudio resalta la necesidad de revisar sistemáticamente las experiencias de la UNA en extensión e investigación para mejorar las políticas universitarias y la práctica de la acción sustantiva. Los proyectos permiten a las personas académicas mejorar su interacción y colaboración con organizaciones sociales, instituciones públicas y pares internacionales. Además, el estudio sugiere la necesidad de un análisis sobre la calidad de la articulación observada, así como la necesidad de revisar las políticas para facilitar una mayor articulación con el sector privado y con otras universidades.

El acervo de información almacenado en el Sistema de Información Académica es una herramienta fundamental para monitorear y mejorar los proyectos y las políticas universitarias que rigen estas actividades. No es un sistema perfecto y debe ser mejorado para efectuar consultas personalizadas. Principalmente, todas las personas académicas deben comprometerse a mantener un registro actualizado y confiable de las actividades desarrolladas en los proyectos. El análisis desarrollado además confirma la importancia de contar con concursos internos para el desarrollo de proyectos de extensión e investigación regionalizados que faciliten la articulación y el abordaje de retos complejos para el desarrollo sostenible y el manejo de los recursos naturales. Sin los concursos internos regionalizados el alcance de las sedes se reduciría drásticamente.

Finalmente, los lineamientos de fondos concursables que promuevan la articulación externa son importantes para contribuir de mejor manera al desarrollo regional. Sin embargo, estos lineamientos deben considerar la simplificación administrativa como estrategia para fomentar la articulación. 
Revista Universidad en DiÁlogo • Vol. 8, N. ${ }^{\circ}$ 1, Enero-Junio, 2018, pp. 39-56

ISSN 2215-2849 • EISSN: 2215-4752

DOI: http://dx.doi.org/10.15359/udre.8-1.3

\section{Referencias}

Asamblea Legislativa (2012). Ley 9036: transforma el Instituto de Desarro1lo Agrario (IDA) en el Instituto de Desarrollo Rural (INDER) y crea Secretaría Técnica de Desarrollo Rural. La Gaceta Digital, (103).

Castro, S. (2009). Costa Rica frente a la regionalización de la educación superior. El primer centro universitario regional en San Ramón, Alajuela. InterSedes: Revista de las Sedes Regionales, X(18), 174203. Recuperado de https://revistas.ucr.ac.cr/index.php/intersedes/ article/view/1033/1094

Consejo Nacional de Rectores (2008). Lineamientos generales para la regionalización universitaria. San José, Costa Rica.

Consejo Nacional de Rectores (2011). Plan Nacional de la Educación Superior Universitaria Estatal 2011-2015. Oficina de Planificación de la Educación Superior. Comisión de Directores de Planificación. Recuperado de http://siesue.conare.ac.cr/images/documentos/investigacion/Conare-PLANES.pdf

Consejo Académico de la Universidad Nacional de Costa Rica (2009). Lineamientos para la gestión de programas, proyectos y actividades. Heredia, Costa Rica. Recuperado de http://www.documentos.una. ac.cr/bitstream/handle/unadocs/1793/Lineamientos\%20para\%20 la $\% 20$ gestion $\% 20 \mathrm{de} \% 20$ programas $\% 20$ proyectos $\% 20 \mathrm{y} \% 20$ actividades\%20academicas.389.pdf? sequence $=1 \&$ isAllowed $=\mathrm{y}$

Consejo Académico de la Universidad Nacional de Costa Rica (2016). Reglamento del Fondo Universitario para el Desarrollo Regional. Gaceta Extraordinaria No. 20. Recuperado de http://www.documentos. una.ac.cr/handle/unadocs/1184

Consejo Académico de la Universidad Nacional de Costa Rica (2017). Reglamento del Fondo Institucional de Desarrollo Académico. $\mathrm{Ga}$ ceta Ordinaria No. 13 Recuperado de http://documentos.una.ac.cr/ handle/unadocs/1183

Fernández, D. (2015). Rezago en carrera académica de personas académicas propietarias de la Universidad Nacional. Universidad en Diálogo: Revista de Extensión, 5(2), 171-186. Recuperado de http://revistas.una.ac.cr/index.php/dialogo/article/view/8440/9499 
Gamboa, R., Céspedes, M., Mena, P. y Azofeifa, C. (2017). La extensión universitaria y los procesos participativos de desarrollo local en Costa Rica. En J. O. Castro y H. Tomassino (eds.), Los caminos de la extensión en América Latina y el Caribe (pp. 53-76). Santa Rosa, Argentina: Universidad Nacional de La Pampa.

Lezcano, S. (2011). Articulación universitaria y desarrollo local: la experiencia del Programa de Regionalización Interuniversitaria de la Región Chorotega, Costa Rica. En Integración, Extensión, Docencia e Investigación para la Inclusión y la Cohesión Social. XI Congreso Iberoamericano de Extensión Universitaria, Santa Fe, Argentina. Recuperado de https://www.unl.edu.ar/iberoextension/dvd/archivos/ponencias/mesa3/articulacion-interuniversita.pdf

Maldonado, T. (2014). Programa Interuniversitario Estatal y desarrollo local en Costa Rica. Universidad en Diálogo: Revista de Extensión, 1(2), 20. Recuperado de http://www.revistas.una.ac.cr/index.php/ dialogo/article/view/5608/5484

Monge, C., Lezcano, S. y Méndez, N. (2014). Generando capacidades regionales mediante la extensión universitaria: experiencia de UNA-Sede Regional Chorotega. Universidad en Diálogo: Revista de Extensión, 1(2), 20. Recuperado de http:/www.revistas.una.ac. cr/index.php/dialogo/article/view/5609/5485

Pilhöfer, A. y Unwin, A. (2013). New approaches in visualization of categorical data: R package extracat. Journal of Statistical Software, 53(7), 1-25. Recuperado de http://10.18637/jss.v053.107

Poder Ejecutivo (2005). Decreto Ejecutivo 32488: de los Comités Sectoriales Regionales Agropecuarios (CSRA) y de los Comités Sectoriales Locales (COSEL), de su Constitución y Funciones. La Gaceta Digital No. 146.

Poder Ejecutivo (2012). Directriz 038: promueve el funcionamiento de los Consejos Cantonales de Coordinación Interinstitucional. La Gaceta Digital No. 188.

QSR International (2017). NVivo 11 Plus for Windows (Version 11-4-11064): QSR International Pty Ltd. Recuperado de https:/www.qsrinternational.com/nvivo/home 
R Development Core Team (2008). R: A language and environment for statistical computing (Version 3.4.1). Vienna, Austria: R Foundation for Statistical Computing. Recuperado de http://www.R-project.org

Rojas, O. (2017). Informe de gestión de la decanatura de la Sede Regional Chorotega. Liberia, Costa Rica: Sede Regional Chorotega-Universidad Nacional de Costa Rica. Recuperado de https://drive.google. com/file/d/0B5hAJ1YV53kvT290NGlrQ2hTRk0/view

Schmidt, I. (2016). La Universidad Nacional de Costa Rica y las sedes regionales como mecanismo de inclusión social, Universidad en Diálogo, 6(2), 11. doi:10.15359/udre.6-2.11

TIMEGRAPHICS (2017). Free online timeline maker. Recuperado de https://time.graphics

Universidad Nacional de Costa Rica (2016). Estatuto Orgánico. Gaceta Extraordinaria $\mathrm{N}^{\circ} .3,80$.

Universidad Nacional de Costa Rica (2017). Reglamento para la Gestión de Programas, Proyectos y Actividades Académicas en la Universidad Nacional. Gaceta Ordinaria No. 5, 81.

Weiss, J. A. (1987). Pathways to Cooperation among public agencies. Journal of Policy Analysis and Management, 7(1), 94-117. doi: $10.2307 / 3323353$

Wickham, H. (2009). Elegant graphics for data analysis. New York, US: Springer.

Zúñiga, S. y Lezcano, S. (2015). Región Chorotega: proyectos y espacios de articulación dirigidos a incidir en la generación de empleo, ingresos, acceso y calidad de agua para consumo humano. Universidad Nacional, Guanacaste, Costa Rica. 